\title{
A Review of Traditional Chinese Medicine in Treating Renal Interstitial Fibrosis via Endoplasmic Reticulum Stress- Mediated Apoptosis
}

\author{
Yu Liu $\mathbb{D}^{1,2}$ Dan-qian Chen $\mathbb{D}^{1},{ }^{1}$ Jing-xue Han $\mathbb{D}^{1,2}$ Ting-ting Zhao $\mathbb{D}^{1},{ }^{1}$ and Shu-ju Li $\mathbb{D}^{2}$ \\ ${ }^{1}$ Beijing Key Lab for Immune-Mediated Inflammatory Diseases, Institute of Clinical Medical Sciences, China- \\ Japan Friendship Hospital, Beijing 100029, China \\ ${ }^{2}$ Heilongjiang Academy of Chinese Medical Sciences, Harbin 150001, China
}

Correspondence should be addressed to Ting-ting Zhao; ttfrfr@163.com and Shu-ju Li; 994864212@qq.com

Received 29 October 2020; Revised 23 April 2021; Accepted 7 May 2021; Published 17 May 2021

Academic Editor: Narsingh Verma

Copyright ( 2021 Yu Liu et al. This is an open access article distributed under the Creative Commons Attribution License, which permits unrestricted use, distribution, and reproduction in any medium, provided the original work is properly cited.

\begin{abstract}
Renal interstitial fibrosis (RIF) is the main pathological manifestation of end-stage renal disease. Recent studies have shown that endoplasmic reticulum (ER) stress is involved in the pathogenesis and development of RIF. Traditional Chinese medicine (TCM), as an effective treatment for kidney diseases, can improve kidney damage by affecting the apoptotic signaling pathway mediated by ER stress. This article reviews the apoptotic pathways mediated by ER stress, including the three major signaling pathways of unfolded protein response, the main functions of the transcription factor C/EBP homologous protein. We also present current research on TCM treatment of RIF, focusing on medicines that regulate ER stress. A new understanding of using TCM to treat kidney disease by regulating ER stress will promote clinical application of Chinese medicine and discovery of new drugs for the treatment of RIF.
\end{abstract}

\section{Introduction}

Renal interstitial fibrosis (RIF) is a common pathologic process in which various kidney diseases progress to end-stage renal disease (ESRD) [1]. The degree of RIF is related to the severity of renal damage $[2,3]$. RIF is characterized by excessive accumulation of extracellular matrix (ECM) in the renal interstitium, which leads to fibroblast hyperplasia, renal interstitial inflammatory cell infiltration, and renal tubular atrophy [4]. The gold standard for assessing RIF is pathologic evaluation after tissue biopsy. Evaluations may include staining with hematoxylin and eosin, Masson trichrome, or sirius red. At the same time, some noninvasive assessments, such as magnetic resonance imaging and ultrasound, are used to determine the extent of RIF through examining the shape of the kidney, renal stiffness, and renal blood flow [5]. Current treatment for RIF is multifactorial, including the use of renin-angiotensin system inhibitors and lipid therapy for blood pressure control, and dietary restrictions. Although these strategies have protective effects on the kidneys, the incidence of ESRD is still high [6].

The pathogenesis of RIF is complicated. Previous studies have made great progress in understanding effector cells, extracellular matrix, and cytokine involvement $[7,8]$. A large number of studies have demonstrated that multiple signal transduction pathways are involved in the process of renal fibrosis. At present, the more plausible pathways include bone morphogenic protein (BMP) [9], platelet-derived growth factor (PDGF) [10], hepatocyte growth factor (HGF) [11], vascular endothelial growth factor (VEGF) [12], TGF- $\beta /$ Smad $[13,14]$, Wnt $[15,16]$, and Hedgehog $[17,18]$. However, the molecular mechanisms of RIF need to be further elucidated. Extensive research on stress responses at the cellular level has found organelle stress is associated with many kidney diseases [19]. An area of intense study is the apoptotic pathway induced by endoplasmic 
reticulum stress (ER stress) and its relationship with RIF [19, 20]. It has been reported that the TGF- $\beta 1 / \mathrm{Smad} 2 / 3$ signaling pathway interacts with ER stress and can regulate the expression of podocyte $\alpha$-smooth muscle actin and synergistically induce podocytes to undergo epithelial-mesenchymal transition [21]. By interfering with the occurrence of ER stress or inhibiting the activity of key enzymes in the apoptosis signaling pathway mediated by ER stress, RIF can be attenuated, suggesting that ER stress is a new fibrosis factor in the complex network of renal fibrosis $[22,23]$.

In China, traditional Chinese medicines (TCMs) have a long history of treating kidney disease and are still used as an alternative therapy for renal disorders. TCM has unique advantages in improving the quality of life and long-term survival of patients [24]. Clinical trials have validated that a large number of Chinese herbal formulas are effective for RIF, with active ingredients, such as tripterygium glycosides [25], resveratrol [26, 27], and astragaloside [28]. TCMs can inhibit ER stress by abating the apoptosis pathway, which may explain how herbal medicines exert their renal protective function in the treatment of RIF $[22,29,30]$. This article reviews recent research on TCM in the treatment of RIF, focusing on the core mechanism of ER stress-mediated apoptosis.

\section{ER Stress, Apoptosis, and RIF}

2.1. Endoplasmic Reticulum and ER Stress. The endoplasmic reticulum (ER) is the largest organelle in the cell cytoplasm. It coordinates at least one-third of protein synthesis, folding, and transporting in the cell, and is important for regulating cell homeostasis [31]. The ER has a strong homeostasis system, and the stability of its internal environment is fundamental for ER function. When abnormal proteins, such as misfolded proteins and unfolded proteins, in the ER cavity are increased or calcium ion balance is changed, ER stress can be induced. Various endogenous and exogenous physiologic and pathologic factors can cause ER stress, such as hypoxia, viral infection, nutritional deficiencies, chemical drugs, free radical attack, calcium ion imbalance, and inflammatory challenges [32]. Three kinds of ER stress are recognized: sterol regulatory element and protein-mediated regulatory response, unfolded protein reaction (UPR), and endoplasmic reticulum overload response [33]. ER stress usually refers to UPR that occurs when misfolded or unfolded proteins in the ER increase and activate the stress signal, which is then transmitted to the nucleus through the ER membrane.

The main function of UPR is to restore ER homeostasis and reduce stimulation of the ER caused by internal and external environmental changes. It is the adaptive response of cells to external stimuli, including reducing protein synthesis, promoting the degradation of abnormal proteins, and participating in the correct folding of proteins [32]. UPR mediates three signaling pathways, which are mainly decomposed by three factors: protein kinase RNA-like endoplasmic reticulum kinase (PERK), activated transcription factor 6 (ATF6), and inositol-requiring enzyme 1 (IRE1) [34]. When the ER is in a steady state, all three sensor proteins are in an inactive state by binding to molecular chaper- one glucose regulatory protein 78/immunoglobulin heavychain-binding protein (GRP78/Bip). When ER homeostasis imbalance induces ER stress, the three receptor proteins separate from GRP78/Bip, leading to activation of receptor proteins. After their activation, PERK, IRE1, and ATF6 activate three signaling pathways: PERK/eIF2 $\alpha / A T F 4$, IRE1/XBP1, and ATF6. GRP78/Bip is the hallmark protein of the ER stress response. Furthermore, ER stress cannot simultaneously activate three UPR signaling branches. Activation of ATF6 and IRE1 occurs immediately and decays over time through an undefined mechanism in ER stress, while PERK is continuously activated $[35,36]$. When the high intensity of ER stress occurs or persists, ER-related molecules cannot restore homeostasis and induce cellular apoptosis [37].

2.2. ER Stress-Mediated Apoptosis Pathway. The ER stressmediated apoptosis signaling pathway is mainly activated through $\mathrm{CHOP}$, JNK, and caspase signaling pathways [38, 39]. The main concern is the CHOP signaling pathway, which can also trigger the caspase signaling pathway. Through the binding site within the CHOP promoter, the UPR signaling pathway leads to the initiation of $\mathrm{CHOP}$ transcription when under chronic or overwhelming ER stress [40]. CHOP is also known as growth arrest and DNA damage-inducible gene 153 (GADD153). It is a member of the C/EBP family and is a special transcription factor that exists in the ER. CHOP can trigger the endogenous apoptosis pathway and promote cell apoptosis by inhibiting the upregulation of BCL-2 [41]. CHOP can also upregulate the expression of TRB3, thus preventing Akt phosphorylation [42-44]. CHOP can also initiate the exogenous apoptosis pathway through DR4 and DR5 [45] and can trigger the ERO1 $\alpha$ IP3R-Ca ${ }^{2+}$-CaMKII pathway, leading to the accumulation of reactive oxygen species (ROS), which are involved in cell apoptosis [46]. The function and specificity of $\mathrm{CHOP}$ are important for the execution of the ER stress-induced apoptosis signaling pathway.

Three signaling pathways mediated by UPR can induce CHOP transcription. The PERK pathway is dominant in CHOP activation. In ER stress, PERK is activated and phosphorylates eukaryotic translation initiation factor $2 \alpha$ (eIF2 $\alpha$ ), which weakens overall protein synthesis. ATF4 is then transferred to the nucleus after eIF $2 \alpha$ activation and transcriptionally upregulates CHOP and many UPR genes that are essential for amino acid metabolism and redox processes [40]. When cells are under stress, PERK upregulates the expression of CHOP to promote apoptosis; the CHOP pathway is the main pathway by which ER stress induces apoptosis $[27,39]$. The activation of IRE1 is similar to that of PERK. Its luminal domain is first dimerized and then transautophosphorylated. Activated IRE1 uses cytoplasmic RNase domain to cut a 26-nucleotide intron from unspliced X-box-binding protein 1 (XBP1) mRNA to produce spliced XBP1(s). IRE1 also mediates the IRE1-dependent decay of selective mRNAs. $\mathrm{XBP} 1(\mathrm{~s})$ enters the nucleus and induces transcription of genes related to protein folding ability and degradation of the ER. Thus, XBP1(s) upregulates CHOP expression [47]. In the ATF6 branch, type II ER localization protein ATF6 is transported to the Golgi apparatus, where it is used by 


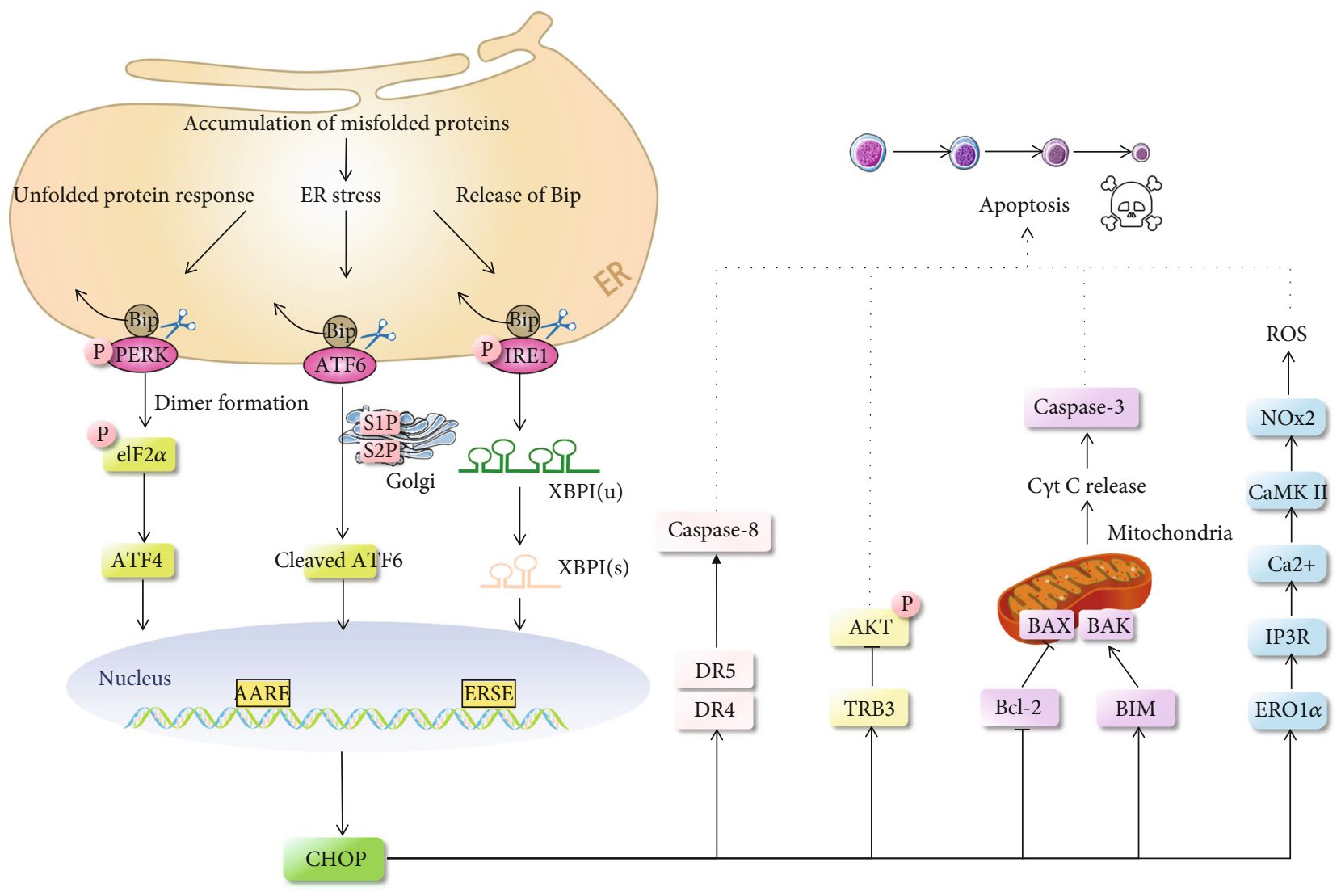

FIGURE 1: Mechanisms of ER stress-mediated apoptosis. Once PERK is activated, eIF2 $\alpha$ is phosphorylated to achieve ATF4 translation and CHOP activation. Activation of the IRE1 $\alpha$ domain processes uncut XBP1mRNA to produce activated XBP1(s), which enters the nucleus and controls the expression of CHOP. ATF $6 \alpha$ is transported to the Golgi apparatus, where it is processed by the proteases SP1 and SP2 to produce cytoplasmic fragment ATF6, which regulates $\mathrm{CHOP}$ activation in the nucleus. CHOP can trigger the endogenous apoptosis pathway and promote cell apoptosis by inhibiting the upregulation of BCL-2. CHOP can also upregulate the expression of TRB3, preventing Akt phosphorylation. CHOP can also initiate the exogenous apoptosis pathway through DR4 and DR5 and can additionally trigger the ERO1 $\alpha$-IP3R-Ca ${ }^{2+}$-CaMKII pathway.

Site- 1 and Site- 2 proteases (SP1 and SP2). In this process, a cytoplasmic fragment of ATF6f is produced and enters the nucleus to regulate expressions of target genes including Bip and CHOP [48] (Figure 1).

2.3. ER Stress-Mediated Apoptosis in RIF. Investigations have confirmed that ER stress is a key factor in renal tubular epithelial cell atrophy and RIF $[49,50]$. The unilateral ureteral obstruction (UUO) model found that the expression of ER stress-related proteins, such as GRP78, CHOP, and caspase-3, is significantly increased [51]. Furthermore, knockout of the CHOP gene not only reduces apoptosis and oxidative stress in experimental renal fibrosis but also reduces local inflammation and attenuates UUO-induced renal fibrosis, indicating that UUO-induced tubular epithelial cell apoptosis is related to the activation of the apoptosis pathway of ER stress [27]. Chronic interstitial nephropathy caused by some drugs is also related to apoptosis of renal tubular epithelial cells. Studies have shown that cyclosporine A can upregulate the expression of apoptosis proteins, such as GRP78, CHOP, and caspase-3, and promote apoptosis of renal tubular epithelial cells through the ER stress pathway [52-54]. This apoptosis is also timeand dose-dependent, and the degree of apoptosis is correlated with the stage of kidney disease [55]. Therefore, the ER stress-mediated apoptosis signaling pathway is one of the important mechanisms of RIF.

\section{Mechanisms of TCMs in Treating RIF by Affecting the ER Stress-Mediated Apoptosis Pathway}

RIF is the result of multiple mechanisms. TCMs have unique advantages in the prevention and treatment of RIF. Multiple compounds in TCMs can intervene in the occurrence and progression of RIF by acting on multiple targets. Traditional herbal formulas have compounds that are rich in antioxidants and minerals, which attenuate oxidative stress/nitrosative stress/ER stress by improving protein folding and cell survival. One such formula is Shenkang injection, which contains the extracts of four medicinal plants: rhubarb root and rhizome (Rheum palmatum L.), astragalus root (Astragalus membranaceus (Fisch.) Bunge), salvia root (Salviae 
miltiorrhizae Bunge), and safflower (Carthamus tinctorius L.) [56]. Studies have found that certain Chinese medicines appear to be effective for RIF by intervening in the apoptosis signaling pathway mediated by ER stress. Single compounds extracted from herbs, whole herbs, and herbal formulas have been investigated (Table 1). In the following, we summarize these investigations.

3.1. Pure Compounds. Resveratrol (RSV) is a natural polyphenol that exists in a variety of edible plants, such as knotweed, grapes, peanuts, and berries. RSV is reported to effectively inhibit obstructive RIF [76]. In the UUO model, RSV can block the expression of eIF $2 \alpha$ and ATF4 proteins, thereby preventing excessive apoptosis of renal tubular epithelial cells and delaying the development of RIF [26]. In diabetic kidney disease (DKD) rats induced by streptozotocin (STZ), RSV treatment has been found to be renal protective by reducing urinary protein level and kidney damage in addition to attenuating ER stress through the PERK pathway [57].

Stachytine is the main biologically active ingredient extracted from the TCM Chinese motherwort (Leonurus sibiricus L.) and has been found to have antifibrotic and antiinflammatory effects [77]. In the UUO model, stachytine not only inhibits the expression and activation of caspase- 12 but also reduces the expression of PERK, CHOP, and caspase- 3 and interferes with the ER stress-mediated apoptosis pathway, eventually inhibiting apoptosis of renal tubular epithelial cells and delaying the occurrence of RIF [58,59].

Emodin, a phenolic compound extracted from rhubarb, knotweed, and other herbs, is widely used to reduce inflammation and inhibit cell proliferation and ER stress $[78,79]$. In KKAy model mice, emodin increased the expression of podocyte nephrin, significantly reduced apoptosis and ER stress markers (GRP78), and also reduced p-PERK, p-eIF2 $\alpha$, ATF4, and CHOP expression. Results of in vitro experiments found that emodin treatment reduced both the level of GRP78 and apoptosis of podocytes cultured with high glucose by inhibiting the upregulation of phosphorylated PERK, P-eIF2 $\alpha$, ATF4, and CHOP. These findings are consistent with the results of animal experiments in which emodin was shown to reduce podocyte apoptosis induced by ER stress through inhibition of the PERK-eIF2 $\alpha$ pathway, downregulation of the expression of $\mathrm{CHOP}$, thus ultimately delaying progression of renal fibrosis [63].

Ginsenoside-Rg1 (G-Rg1) is the main active component in ginseng (Panax ginseng C. A. Mey). G-Rg1 has antioxidative, antiproliferation, and antiapoptosis effects and can significantly inhibit organ fibrosis [80]. G-Rg1 has been shown to inhibit excessive apoptosis of renal tubular epithelial cells and delay progression of renal fibrosis by reducing expressions of GRP78 and its downstream proapoptotic factors CHOP and caspase-12 [51]. In addition, G-Rg1 treatment in chronic CsA nephropathy models can reduce expressions of GRP78 and CHOP and can inhibit apoptosis of renal tubular cells triggered by ER stress [60].

Astragaloside IV (AS-IV) is extracted from the Chinese herbal medicine astragalus (Astragalus membranaceus (Fisch.) Bunge), which is widely used to treat diabetic nephropathy and other kidney diseases in China. AS-IV treatment can prevent STZ-induced rat mesangial matrix expansion. It can significantly inhibit phosphorylation of eIF $2 \alpha$, PERK, and JNK both in vivo and in vitro and significantly inhibit expressions of GRP78 and ORP150 and prevent podocyte apoptosis induced by tunicamycin in vitro, while reducing the expression of CHOP and caspase-3 [29]. Another study came to the same conclusion that in the STZ-induced rat, AS-IV has a protective effect on ER stress-induced podocyte apoptosis by inhibiting the PERKATF4-CHOP pathway [61]. Furthermore, AS-IV treatment can inhibit apoptosis of renal tubular epithelial cells induced by ER stress by downregulating expressions of p-PERK, ATF4, and CHOP [62].

Curcumin is a natural polyphenol compound present in the rhizome of turmeric (Curcuma longa L.). Curcumin has anti-inflammatory, antioxidant, and antiapoptotic properties and has excellent safety [81]. Curcumin has been shown to effectively prevent and treat renal fibrosis by attenuating the increased protein expression of mitogen-activated protein kinases (MAPKs), such asp-JNK, p-ERK1/2, GRP78, and $\mathrm{CHOP}$ in the kidney tissue of nonalcoholic steatohepatitis (NASH) mice [82]. In addition, curcumin also reduced NASH renal cell apoptosis signaling protein (cleaved caspase-3, cleaved caspase-12). These results indicate that curcumin protects against the development of chronic kidney disease in NASH mice by reducing ER stress-induced apoptosis and MAPK signal transduction [30].

Natural flavonoid morin hydrate $(\mathrm{MH})$ is a compound isolated from white mulberry (Morus alba $\mathrm{L}$.) and other fruits such as apple (Malus), Osage orange (Maclura pomifera), guava (Psidium guajava), and fig (Ficus carica). MH has a wide range of pharmacologic activities, including antioxidant, anti-inflammatory, antiapoptotic, and antiautophagy [83-85]. In cisplatin-treated HEK-293 cells and mouse kidneys, ER stress was induced through increasing intracellular ROS and decreasing antioxidant enzymes. After exposure to $\mathrm{MH}$, ER stress markers, such as the expression of PERK, IRE- $1 \alpha$, p-eIF $2 \alpha$, CHOP, and casp-12, were all attenuated. Furthermore, MH may inhibit the mechanism of autophagy and cell death mediated by ER stress, indicating that $\mathrm{MH}$ protects against cisplatin-treated kidney damage [64].

Withaferin A (WA) is a compound in ashwagandha (Withania somnifera) that has antioxidant and anticancer activities [86]. Ashwagandha is commonly used in India as a traditional medicine and dietary supplement [87]. Studies have demonstrated that WA improves symptoms of several chronic diseases and cancers $[88,89]$. In laboratory mice, WA significantly reduced histopathologic changes and collagen deposition of UUO in the kidneys and reduced UUO mouse endoplasmic reticulum stress-related molecules (GRP78, GRP94, ATF4, CHOP, phosphorylated eIF2 $\alpha$, and cleaved caspase-12). Thus, WA is able to protect against the progression of chronic kidney disease by improving endoplasmic reticulum stress-related apoptosis, inflammation, and fibrosis [65].

3.2. Extracts. Cordyceps sobolifera (CS) is a TCM that has long been used to treat kidney diseases. Water extract of 


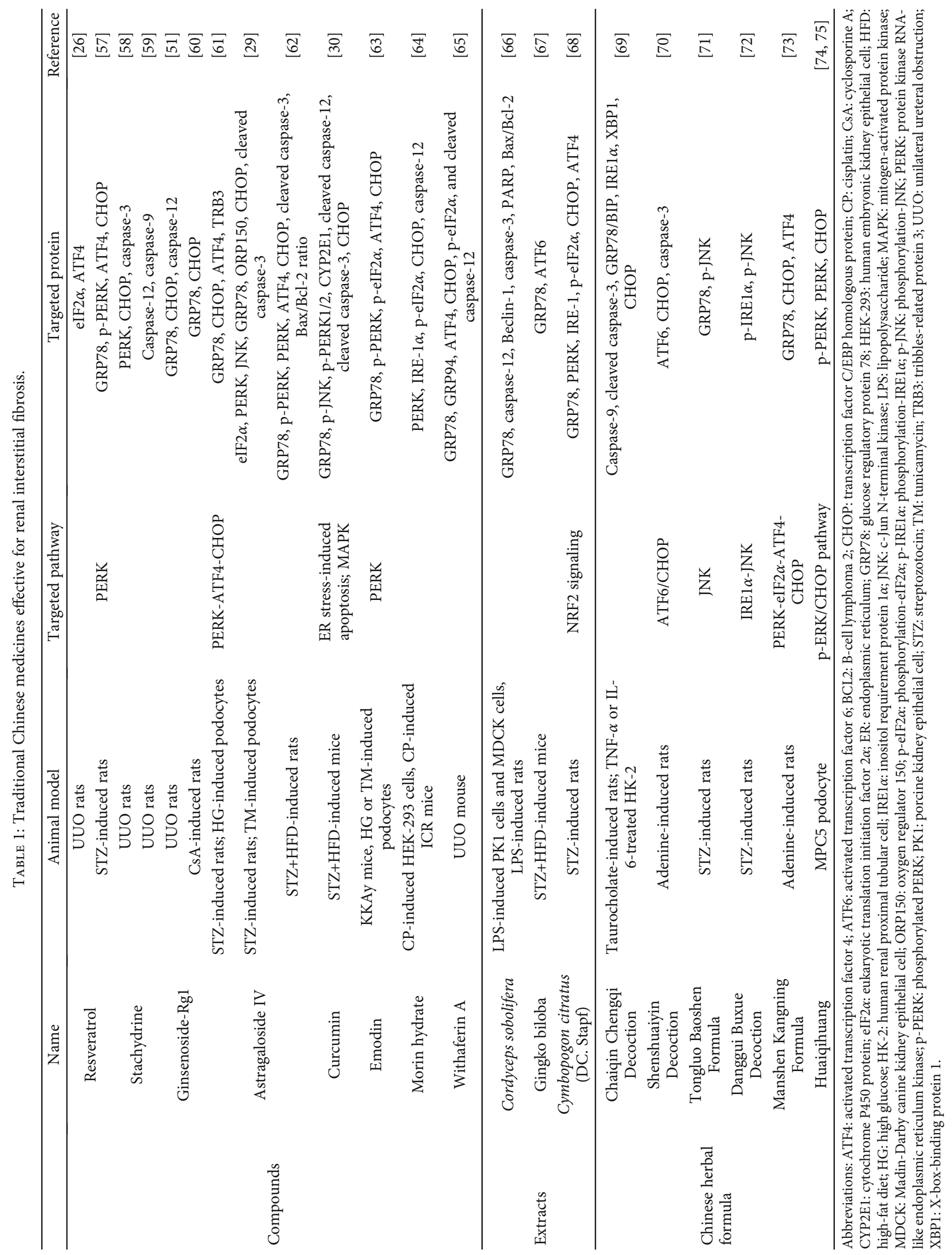


fermented decoction of CS can attenuate lipopolysaccharideinduced ER stress and tissue damage and reduce cell apoptosis through reducing GRP78, caspase-12, Bax/Bcl-2 ratio, and caspase-3 expression [66].

Ginkgo biloba extract (GBE) is derived from ginkgo leaf (Ginkgo biloba L.). Its main active ingredients are flavonoids and terpenoid lactones. GBE has a wide range of clinical applications and is mostly used in the treatment of cardiovascular and cerebrovascular diseases [90]. In DKD mice induced by STZ and high-fat diet, the expression of ER stress markers GRP78 and ATF6 in renal tissue decreased after GBE treatment. Moreover, GBE was found to reduce urine $\beta 2-M G, R B P 4$, and NGAL levels in mice, indicating that GBE attenuates renal tubular damage in DKD mice [67].

Lemongrass (Cymbopogon citratus (DC.) Stapf) is commonly used in traditional medicine for the management of a number of diseases including diabetes mellitus [91, 92]. Methanolic extract of lemongrass leaves was found to reduce ER stress induced by streptozotocin in rats by downregulating GRP78 and upregulating Nrf2 signaling. This may be the effects of the flavonoids apigenin, quercetin, and kaempferol that are found in lemongrass [68].

3.3. Chinese Herbal Formulas. Several Chinese herbal formulas are known to treat RIF through inhibiting ER stress. Chaiqin Chengqi Decoction is comprised of the following TCMs: bupleurum root (Bupleurum chinense DC.), scutellaria root (Scutellaria baicalensis Georgi), rhubarb root and rhizome (Rheum palmatum L.), crystallized sodium sulfate, magnolia bark (Magnolia officinalis Rehder \& E.H.Wilson), bitter orange (Citrus aurantium L.), virgate wormwood (Artemisia scoparia Waldst. \& Kitam.), and gardenia fruit (Gardenia jasminoides J.Ellis). In an animal model of acute kidney injury caused by acute pancreatitis, Chaiqin Chengqi Decoction was found to inhibit renal ER stress indicators (BIP, XBP1s, and CHOP) and apoptotic protein caspases (caspase- 9 and Cle-caspase-3) and reduce pathophysiologic changes and cell apoptosis in the kidney, thereby restoring kidney function. In vitro experiments found that both TNF- $\alpha$ and IL- 6 activate ER stress of HK-2 cells. Chaiqin Chengqi Decoction can restore cellular ER stress indicators and the expression level of caspases and reduce the number of dead HK-2 cells [69].

Shenshuaiyin Decoction consists of the following TCMs: astragalus root (Astragalus membranaceus (Fisch.) Bunge), prince's feather (Polygonum orientale L.), pseudostellaria root (Pseudostellaria heterophylla (Miq.) Pax), self-heal spike (Prunella vulgaris L.), round cardamom (Amomum kwangsiense D. Fang \& X.X.Chen), white atractylodes rhizome (Atractylodes macrocephala Koidz.), cuscuta seed (Cuscuta chinensis Lam.), smooth greenbrier rhizome (Smilax glabra Roxb.), cogon grass root (Imperata koenigii (Retz.) P. Beauv.), agastache (Agastache rugosa (Fisch. \& C.A.Mey.) Kuntze), rhubarb (Rheum palmatum L.), pinellia tuber (Pinellia ternata (Thunb.) Breit.), salvia root (Salvia miltiorrhiza Bunge), leech (Hirudo nipponica Whitman), curcuma rhizome (Curcuma zedoaria (Christm.) Roscoe), and Chinese soft-shelled turtle shell (Trionyx sinensis Wiegmann). Shenshuaiyin Decoction has been found to reduce kidney damage and improve kidney function. In the adenine-induced renal failure rat model, treatment with Shenshuaiyin Decoction attenuated expression levels of ATF6, CHOP, and caspase-3 protein in kidney tissues, indicating that this medicine may protect kidney function by affecting the ATF6/CHOP pathway and reducing renal cell apoptosis [70].

Tongluo Baoshen Formula is comprised of the following TCMs: astragalus root (Astragalus membranaceus (Fisch.) Bunge), cornus fruit (Cornus officinalis Sieb. et Zucc.), privet fruit (Ligustrum lucidum W.T.Aiton), salvia root (Salviae miltiorrhizae Bunge), chuanxiong root (Ligusticum chuanxiong Hort.), leech (Hirudo nipponica Whitman), cogongrass (Imperata cylindrica (L.) P. Beauv.), plantago seed (Plantago asiatica L.), and rhubarb (Rheum palmatum L.). Research has shown that Tongluo Baoshen Formula can significantly reduce the expression of GRP78 and p-JNK protein in the kidney tissue of diabetic rats, inhibit ER stress response and its induced JNK apoptosis signaling pathway, reduce renal cell apoptosis, and protect kidney function [71].

Danggui Buxue Decoction is a simple formula that has only two TCMs: astragalus root (Astragalus membranaceus (Fisch.) Bunge) and tangkuei (Angelica sinensis (Oliv.) Diels). In the STZ-induced diabetic rat model, Danggui Buxue Decoction treatment reduced expressions of $\mathrm{p}$-IRE $1 \alpha$ and $\mathrm{p}$-JNK protein as well as apoptosis in kidney tissue, thus lessening the ER stress response of the kidney under high glucose condition and protecting the kidney [72].

Manshen Kangning Formula is yet another Chinese herbal prescription that treats RIF. The formula consists of the following TCMs: astragalus root (Astragalus membranaceus (Fisch.) Bunge), codonopsis root (Codonopsis pilosula (Franch.) Nannf.), poria (Poria cocos (Schw.) Wolf), epimedium aerial parts (Epidemium), cornus fruit (Cornus officinalis Sieb. et Zucc.), ophiopogon tuber (Ophiopogon japonicas (Thunb.) Ker-Gawl.), rehmannia root (Rehmannia glutinosa Libosch), salvia root (Salviae miltiorrhizae Bunge), cuttlefish bone (Sepiella maindroni Rochebruneor Sepia esculenta Hoyle), and rhubarb root and rhizome (Rheum palmatum L.). In the adenine-induced renal failure rat model, Manshen Kangning Formula reduced apoptosis in kidney tissue and expressions of GRP78, GRP78mRNA, ATF-4mRNA, and CHOPmRNA. Therefore, the formula has an inhibitory effect on the overexpression of the PERK-eIF2 $\alpha$-ATF4$\mathrm{CHOP}$ pathway, thus attenuating the ER stress response and protecting renal function [73].

Finally, another Chinese formula that has been studied for its ability to treat nephropathy is Huaiqihuang. The formula consists of the fungus trametes, goji berry (Lycium barbarum L.), and polygonatum rhizome (Polygonatum sibiricum F.Delaroche), which have long been used in China to treat kidney disease. Research has shown that mechanisms by which they exert their effects include regulating oxidative stress and inhibiting apoptosis [93]. Other studies have shown that the formula protects podocytes by suppressing the $\mathrm{p}$-ERK/CHOP signaling pathway and reversing mercury-induced upregulation of GRP78 and reducing dysfunction of podocyte apoptosis and DNA damage [74, 75]. Thus, Huaiqihuang inhibits cell apoptosis by inhibiting ER stress and has a therapeutic effect on podocyte dysfunctionrelated renal diseases. 


\section{Discussion and Conclusion}

Traditional Chinese medicines (TCMs) have been used effectively in China for centuries to treat and delay progression of kidney diseases. The therapeutic value of TCMs and their isolated compounds in the treatment of RIF has been demonstrated by a large number of animal experiments based on modern pharmacologic research. However, clinical application and research on TCMs aimed at regulating ER stress are limited; therefore, well-designed and executed clinical studies are needed. Although the TCMs described above are not toxic at normal therapeutic doses, we must be aware that some TCMs may cause significant toxicity, including nephrotoxicity. Biologically active compounds extracted from those TCMs may actually promote excessive ER stress, which may be the important mechanism of their toxicity. To date, most studies on TCMs have shown their protective effect against ER stress in the kidneys. However, further exploration is needed to clarify how TCMs reduce ER stress in renal interstitial fibrosis and thus cell apoptosis.

In this review, we mainly discussed the potential mechanisms of TCMs in the treatment of RIF by regulating cell apoptosis caused by ER stress. Chinese herbal formulas such as Chaiqin Chengqi Decoction, Shenshuaiyin Decoction, Tongluo Baoshen Formula, and various active compounds extracted from TCMs, such as resveratrol, astragaloside IV, and emodin are effective for the prevention and treatment of RIF. However, in-depth investigations are needed to determine the mechanisms of Chinese medicines in regulating ER stress, which may lead to new treatment strategies for RIF.

\section{Conflicts of Interest}

The authors declare that there is no conflict of interest regarding the publication of this paper.

\section{Acknowledgments}

We would like to thank Nissi S. Wang, MSc, for the developmental editing of this manuscript. This study was supported by the National Natural Scientific Foundation of China (No. 81973627).

\section{References}

[1] H. H. Szeto, "Pharmacologic approaches to improve mitochondrial function in AKI and CKD," Journal of the American Society of Nephrology, vol. 28, no. 10, pp. 2856-2865, 2017.

[2] B. C. Liu, T. T. Tang, L. L. Lv, and H. Y. Lan, "Renal tubule injury: a driving force toward chronic kidney disease," Kidney International, vol. 93, no. 3, pp. 568-579, 2018.

[3] B. C. Liu, T. T. Tang, and L. L. Lv, "How tubular epithelial cell injury contributes to renal fibrosis," Advances in Experimental Medicine and Biology, vol. 1165, pp. 233-252, 2019.

[4] B. D. Humphreys, "Mechanisms of renal fibrosis," Annual Review of Physiology, vol. 80, no. 1, pp. 309-326, 2018.

[5] K. Jiang, C. M. Ferguson, and L. O. Lerman, "Noninvasive assessment of renal fibrosis by magnetic resonance imaging and ultrasound techniques," Translational Research, vol. 209, pp. 105-120, 2019.
[6] M. Ruiz-Ortega, S. Rayego-Mateos, S. Lamas, A. Ortiz, and R. R. Rodrigues-Diez, "Targeting the progression of chronic kidney disease," Nature Reviews. Nephrology, vol. 16, no. 5, pp. 269-288, 2020.

[7] A. B. Farris and R. B. Colvin, "Renal interstitial fibrosis: mechanisms and evaluation," Current Opinion in Nephrology and Hypertension, vol. 21, no. 3, pp. 289-300, 2012.

[8] S. Djudjaj and P. Boor, "Cellular and molecular mechanisms of kidney fibrosis," Molecular Aspects of Medicine, vol. 65, pp. 16-36, 2019.

[9] S. Kim, C. H. Jeong, S. H. Song et al., "Micellized protein transduction domain-bone morphogenetic protein-7 efficiently blocks renal fibrosis via inhibition of transforming growth factor-beta-mediated epithelial-mesenchymal transition," Frontiers in Pharmacology, vol. 11, p. 591275, 2020.

[10] H. M. Kok, L. L. Falke, R. Goldschmeding, and T. Q. Nguyen, "Targeting CTGF, EGF and PDGF pathways to prevent progression of kidney disease," Nature Reviews. Nephrology, vol. 10, no. 12, pp. 700-711, 2014.

[11] F. Yang, L. Deng, J. Li et al., "Emodin retarded renal fibrosis through regulating HGF and TGFbeta-Smad signaling pathway," Drug Design, Development and Therapy, vol. Volume 14, pp. 3567-3575, 2020.

[12] K. Tanabe, J. Wada, and Y. Sato, “Targeting angiogenesis and lymphangiogenesis in kidney disease," Nature Reviews. Nephrology, vol. 16, no. 5, pp. 289-303, 2020.

[13] Y. Y. Gu, X. S. Liu, X. R. Huang, X. Q. Yu, and H. Y. Lan, "Diverse role of TGF- $\beta$ in kidney disease," Frontiers in Cell and Development Biology, vol. 8, p. 123, 2020.

[14] X.-M. Meng, P. M.-K. Tang, J. Li, and H. Y. Lan, "TGF-beta/Smad signaling in renal fibrosis," Frontiers in Physiology, vol. 6, p. 82, 2015.

[15] O. H. Maarouf, A. Aravamudhan, D. Rangarajan et al., "Paracrine Wnt1 drives interstitial fibrosis without inflammation by tubulointerstitial cross-talk," J Am Soc Nephrol, vol. 27, no. 3, pp. 781-790, 2016.

[16] T. Kawakami, S. Ren, and J. S. Duffield, "Wnt signalling in kidney diseases: dual roles in renal injury and repair," The Journal of Pathology, vol. 229, no. 2, pp. 221-231, 2013.

[17] D. Zhou, R. J. Tan, and Y. Liu, "Sonic hedgehog signaling in kidney fibrosis: a master communicator," Science China. Life Sciences, vol. 59, no. 9, pp. 920-929, 2016.

[18] D. Zhou, Y. Li, L. Zhou et al., "Sonic hedgehog is a novel tubule-derived growth factor for interstitial fibroblasts after kidney injury," J Am Soc Nephrol, vol. 25, no. 10, pp. 21872200, 2014.

[19] A. V. Cybulsky, "Endoplasmic reticulum stress, the unfolded protein response and autophagy in kidney diseases," Nature Reviews Nephrology, vol. 13, no. 11, pp. 681-696, 2017.

[20] S. A. Oakes and F. R. Papa, "The role of endoplasmic reticulum stress in human pathology," Annual Review of Pathology, vol. 10, no. 1, pp. 173-194, 2015.

[21] C. A. Chen, J. M. Chang, E. E. Chang, H. C. Chen, and Y. L. Yang, "Crosstalk between transforming growth factor- $\beta 1$ and endoplasmic reticulum stress regulates alpha-smooth muscle cell actin expression in podocytes," Life Sciences, vol. 209, pp. 9-14, 2018.

[22] B. Ke, N. Zhu, F. Luo, Y. Xu, and X. Fang, "Targeted inhibition of endoplasmic reticulum stress: new hope for renal fibrosis (review)," Molecular Medicine Reports, vol. 16, no. 2, pp. 1014-1020, 2017. 
[23] L. Ling, M. Yang, W. Ding, and Y. Gu, "Ghrelin attenuates UUO-induced renal fibrosis via attenuation of Nlrp3 inflammasome and endoplasmic reticulum stress," American Journal of Translational Research, vol. 11, no. 1, pp. 131-141, 2019.

[24] K. C. Huang, Y. C. Su, M. F. Sun, and S. T. Huang, "Chinese herbal medicine improves the long-term survival rate of patients with chronic kidney disease in Taiwan: a nationwide retrospective population-based cohort study," Frontiers in Pharmacology, vol. 9, p. 1117, 2018.

[25] X. W. Wang, R. M. Tian, Y. Q. Yang et al., “Tripterygium glycoside fraction $\mathrm{n} 2$ ameliorates adriamycin-induced nephrotic syndrome in rats by suppressing apoptosis," Journal of Ethnopharmacology, vol. 257, p. 112789, 2020.

[26] C. Zhang, Y. Zhou, Y. Zhou, Y. Lu, and D. Wang, "Regulation of eIF $2 \alpha$ expression and renal interstitial fibrosis by resveratrol in rat renal tissue after unilateral ureteral obstruction," Renal Failure, vol. 38, no. 4, pp. 622-628, 2016.

[27] S.-H. Liu, C.-T. Wu, K.-H. Huang et al., "C/EBP homologous protein (CHOP) deficiency ameliorates renal fibrosis in unilateral ureteral obstructive kidney disease," Oncotarget, vol. 7, no. 16, pp. 21900-21912, 2016.

[28] X. Zhou, X. Sun, X. Gong et al., “Astragaloside IV from Astragalus membranaceus ameliorates renal interstitial interstitial by inhibiting inflammation via TLR4/NF-кB in vivo and in vitro," International Immunopharmacology, vol. 42, pp. 18-24, 2017.

[29] Z. S. Wang, F. Xiong, X. H. Xie, D. Chen, J. H. Pan, and L. Cheng, "Astragaloside IV attenuates proteinuria in streptozotocin-induced diabetic nephropathy via the inhibition of endoplasmic reticulum stress," BMC Nephrology, vol. 16, no. 1, p. 44, 2015.

[30] M. R. Afrin, S. Arumugam, M. A. Rahman et al., "Curcumin reduces the risk of chronic kidney damage in mice with nonalcoholic steatohepatitis by modulating endoplasmic reticulum stress and MAPK signaling," International Immunopharmacology, vol. 49, pp. 161-167, 2017.

[31] D. S. Schwarz and M. D. Blower, "The endoplasmic reticulum: structure, function and response to cellular signaling," Cellular and Molecular Life Sciences, vol. 73, no. 1, pp. 79-94, 2016.

[32] M. Wang and R. J. Kaufman, "Protein misfolding in the endoplasmic reticulum as a conduit to human disease," Nature, vol. 529, no. 7586, pp. 326-335, 2016.

[33] H. L. Pahl, "Signal transduction from the endoplasmic reticulum to the cell nucleus," Physiological Reviews, vol. 79, no. 3, pp. 683-701, 1999.

[34] S. S. Cao and R. J. Kaufman, "Endoplasmic reticulum stress and oxidative stress in cell fate decision and human disease," Antioxidants \& Redox Signaling, vol. 21, no. 3, pp. 396-413, 2014.

[35] D. T. Rutkowski, S. M. Arnold, C. N. Miller et al., "Adaptation to ER Stress Is Mediated by Differential Stabilities of ProSurvival and Pro-Apoptotic mRNAs and Proteins," PLoS Biology, vol. 4, no. 11, p. e374, 2006.

[36] J. H. Lin, H. Li, D. Yasumura et al., "IRE1 Signaling Affects Cell Fate During the Unfolded Protein Response," Science, vol. 318, no. 5852, pp. 944-949, 2007.

[37] C. Hetz, J. M. Axten, and J. B. Patterson, "Pharmacological targeting of the unfolded protein response for disease intervention," Nature Chemical Biology, vol. 15, no. 8, pp. 764-775, 2019.

[38] C. Hetz, "The unfolded protein response: controlling cell fate decisions under ER stress and beyond," Nature Reviews. Molecular Cell Biology, vol. 13, no. 2, pp. 89-102, 2012.
[39] S. Oyadomari and M. Mori, "Roles of CHOP/GADD153 in endoplasmic reticulum stress," Cell Death and Differentiation, vol. 11, no. 4, pp. 381-389, 2004.

[40] W. Rozpedek, D. Pytel, B. Mucha, H. Leszczynska, J. A. Diehl, and I. Majsterek, "The role of the PERK/eIF2 $\alpha /$ ATF4/CHOP signaling pathway in tumor progression during endoplasmic reticulum stress," Current Molecular Medicine, vol. 16, no. 6, pp. 533-544, 2016.

[41] M. A. Carpio, M. Michaud, W. Zhou, J. K. Fisher, L. D. Walensky, and S. G. Katz, "BCL-2 family member BOK promotes apoptosis in response to endoplasmic reticulum stress," Proceedings of the National Academy of Sciences of the United States of America, vol. 112, no. 23, pp. 72017206, 2015.

[42] K. Du, "TRB3: A tribbles Homolog That Inhibits Akt/PKB Activation by Insulin in Liver," Science, vol. 300, no. 5625, pp. 1574-1577, 2003.

[43] W. Wang, J. Cheng, A. Sun et al., “TRB3 mediates renal tubular cell apoptosis associated with proteinuria," Clinical and Experimental Medicine, vol. 15, no. 2, pp. 167-177, 2015.

[44] Y. Ma, F. Chen, S. Yang, Y. Duan, Z. Sun, and J. Shi, "Silencing of TRB3 ameliorates diabetic tubule interstitial nephropathy via PI3K/AKT signaling in rats," Medical Science Monitor, vol. 23, pp. 2816-2824, 2017.

[45] P. Chen, T. Hu, Y. Liang et al., "Neddylation Inhibition Activates the Extrinsic Apoptosis Pathway through ATF4CHOP-DR5 Axis in Human Esophageal Cancer Cells," Clinical Cancer Research, vol. 22, no. 16, pp. 4144-4157, 2016.

[46] G. Li, M. Mongillo, K. T. Chin et al., "Role of ERO1-alphamediated stimulation of inositol 1,4,5-triphosphate receptor activity in endoplasmic reticulum stress-induced apoptosis," The Journal of Cell Biology, vol. 186, no. 6, pp. 783-792, 2009.

[47] A. H. Lee, N. N. Iwakoshi, and L. H. Glimcher, "XBP-1 Regulates a Subset of Endoplasmic Reticulum Resident Chaperone Genes in the Unfolded Protein Response," Molecular and Cellular Biology, vol. 23, no. 21, pp. 7448-7459, 2003.

[48] J. Ye, R. B. Rawson, R. Komuro et al., "ER stress induces cleavage of membrane-bound ATF6 by the same proteases that process SREBPs," Molecular Cell, vol. 6, no. 6, pp. 1355-1364, 2000.

[49] R. Inagi, "Endoplasmic reticulum stress in the kidney as a novel mediator of kidney injury," Nephron. Experimental Nephrology, vol. 112, no. 1, pp. e1-e9, 2009.

[50] H. Maekawa and R. Inagi, "Stress Signal Network between Hypoxia and ER Stress in Chronic Kidney Disease," Frontiers in Physiology, vol. 8, p. 74, 2017.

[51] S.-s. Li, J.-m. Ye, Z.-y. Deng, L. X. Yu, X. X. Gu, and Q. F. Liu, "Ginsenoside-Rg1 inhibits endoplasmic reticulum stressinduced apoptosis after unilateral ureteral obstruction in rats," Renal Failure, vol. 37, no. 5, pp. 890-895, 2015.

[52] N. Pallet, N. Bouvier, A. Bendjallabah et al., "Cyclosporineinduced endoplasmic reticulum stress triggers tubular phenotypic changes and death," American Journal of Transplantation, vol. 8, no. 11, pp. 2283-2296, 2008.

[53] Y.-M. Choi, H.-Y. Cho, M. A. Anwar, H. K. Kim, J. W. Kwon, and S. Choi, "ATF3 attenuates cyclosporin A-induced nephrotoxicity by downregulating CHOP in HK-2 cells," Biochemical and Biophysical Research Communications, vol. 448, no. 2, pp. 182-188, 2014.

[54] C.-H. Cheng, K.-H. Shu, H.-R. Chang, and M. C. Chou, "Cyclosporine-induced tubular vacuolization: the role of 
Bip/Grp78," Nephron. Experimental Nephrology, vol. 122, no. 1-2, pp. 1-12, 2012.

[55] S. W. Han, C. Li, K. O. Ahn et al., "Prolonged endoplasmic reticulum stress induces apoptotic cell death in an experimental model of chronic cyclosporine nephropathy," American Journal of Nephrology, vol. 28, no. 5, pp. 707-714, 2008.

[56] J.-J. Zou, X.-T. Zhou, Y.-K. Chen et al., "A review on the efficacy and mechanism of action of Shenkang injection against chronic kidney disease," Biomedicine \& Pharmacotherapy, vol. 132, p. 110833, 2020.

[57] D. Yuan, X.-M. Liu, Z. Fang, L.-L. Du, J. Chang, and S.-H. Lin, "Protective effect of resveratrol on kidney in rats with diabetic nephropathy and its effect on endoplasmic reticulum stress," European Review for Medical and Pharmacological Sciences, vol. 22, no. 5, pp. 1485-1493, 2018.

[58] C. Zhang, Y. Lu, Q. Q. Tong et al., "Effect of stachydrine on endoplasmic reticulum stress-induced apoptosis in rat kidney after unilateral ureteral obstruction," Journal of Asian Natural Products Research, vol. 15, no. 4, pp. 373-381, 2013.

[59] C. Zhang, Y. Lu, Y. J. Zhou, Q. Q. Tong, C. Qu, and T. J. Kang, "The effect of stachydrine on the expression of caspase-12 in rats with unilateral ureteral obstruction," The Journal of Urology, vol. 192, no. 5, pp. 1549-1554, 2014.

[60] Q. F. Liu, Z. Y. Deng, J. M. Ye, A. L. He, and S. S. Li, “Ginsenoside Rg1 protects chronic cyclosporin a nephropathy from tubular cell apoptosis by inhibiting endoplasmic reticulum stress in rats," Transplantation Proceedings, vol. 47, no. 2, pp. 566-569, 2015.

[61] Y. Chen, D. Gui, J. Chen, D. He, Y. Luo, and N. Wang, "Downregulation of PERK-ATF4-CHOP pathway by astragaloside IV is associated with the inhibition of endoplasmic reticulum stress-induced podocyte apoptosis in diabetic rats," Cellular Physiology and Biochemistry, vol. 33, no. 6, pp. 1975-1987, 2014.

[62] Y. Ju, Y. Su, Q. Chen et al., "Protective effects of astragaloside IV on endoplasmic reticulum stress-induced renal tubular epithelial cells apoptosis in type 2 diabetic nephropathy rats," Biomedicine \& Pharmacotherapy, vol. 109, pp. 84-92, 2019.

[63] N. Tian, Y. Gao, X. Wang et al., "Emodin mitigates podocytes apoptosis induced by endoplasmic reticulum stress through the inhibition of the PERK pathway in diabetic nephropathy," Drug Design, Development and Therapy, vol. Volume 12, pp. 2195-2211, 2018.

[64] M. P. Singh, A. K. Chauhan, and S. C. Kang, "Morin hydrate ameliorates cisplatin-induced ER stress, inflammation and autophagy in HEK-293 cells and mice kidney via PARP-1 regulation," International Immunopharmacology, vol. 56, pp. 156-167, 2018.

[65] C.-M. Chen, Y.-P. Chung, C.-H. Liu et al., "Withaferin A protects against endoplasmic reticulum stress-associated apoptosis, inflammation, and fibrosis in the kidney of a mouse model of unilateral ureteral obstruction," Phytomedicine, vol. 79, p. 153352, 2020.

[66] M. F. Wu, P. C. Li, C. C. Chen, S. S. Ye, C. T. Chien, and C. C. $\mathrm{Yu}$, "Cordyceps sobolifera extract ameliorates lipopolysaccharide-induced renal dysfunction in the rat," The American Journal of Chinese Medicine, vol. 39, no. 3, pp. 523-535, 2011.

[67] X. Pang, X. Shi, Y. Zhang, J. Han, and X. Sun, "Effect of Ginkgo leaves extract EGB761 on renal tubular injury and endoplasmic reticulum stress in mice with diabetic kidney disease,"
Traditional Chinese Drug Research \& Clinical Pharmacology, vol. 31, no. 8, pp. 879-886, 2020.

[68] O. O. Elekofehinti, A. T. Onunkun, and T. M. Olaleye, "Cymbopogon citratus (DC.) Stapf mitigates ER-stress induced by streptozotocin in rats via down-regulation of GRP78 and upregulation of Nrf2 signaling," Journal of Ethnopharmacology, vol. 262, p. 113130, 2020.

[69] X. Yang, X. Zhang, Z. Lin et al., "Chaiqin chengqi decoction alleviates severe acute pancreatitis associated acute kidney injury by inhibiting endoplasmic reticulum stress and subsequent apoptosis," Biomedicine \& Pharmacotherapy, vol. 125, p. 110024, 2020.

[70] Y. Liu and X. Ma, "Effects of Shenshuaiyin decoction on ATF6/CHOP pathway in kidney tissues of chronic renal failure rats," JETCM, vol. 1, no. 26, pp. 760-770, 2017.

[71] J. Zhang, X.-L. Rong, J.-M. Li et al., "Effects of Tongluo Baoshen formula on apoptosis signaling pathways JNK, Caspase-12 mediated by endoplasmic reticulum stress," CJTCM, vol. 32, no. 11, pp. 5109-5112, 2017.

[72] Y. Shuai, S. Zhang, X. Shen, and Y. Zhang, "Effect of astragals and Angelica mixture on expression of IRE1 $\alpha$-JNK pathway in diabetic nephropathy rats," CHINESE ARCHIVES OF TRADITIONAL CHINESE MEDICINE, vol. 36, no. 6, 2018.

[73] S. Sun, X. Jin, S. Huang et al., "Effect of ManshenKangning formula on PERK/ATF4 pathway in endoplasmic reticulum stress in renal interstitial fibrosis rats," Journal of Traditional Chinese Medicine, vol. 54, no. 3, 2013.

[74] T. Li, J. Mao, L. Huang et al., "Huaiqihuang may protect from proteinuria by resisting MPC5 podocyte damage via targeting p-ERK/CHOP pathway," Bosnian Journal of Basic Medical Sciences, vol. 16, no. 3, pp. 193-200, 2016.

[75] T. X. Li, J. H. Mao, L. Huang et al., "Beneficial effects of Huaiqihuang on hyperglycemia-induced MPC5 podocyte dysfunction through the suppression of mitochondrial dysfunction and endoplasmic reticulum stress," Molecular Medicine Reports, vol. 16, no. 2, pp. 1465-1471, 2017.

[76] D. J. Den Hartogh and E. Tsiani, "Health Benefits of Resveratrol in Kidney Disease: Evidence from In Vitro and In Vivo Studies," Nutrients, vol. 11, no. 7, p. 1624, 2019.

[77] F. Cheng, Y. Zhou, M. Wang et al., "A review of pharmacological and pharmacokinetic properties of stachydrine," Pharmacological Research, vol. 155, p. 104755, 2020.

[78] J. Gao, F. Wang, W. Wang, Z. Su, C. Guo, and S. Cao, "Emodin Suppresses Hyperglycemia-Induced Proliferation and Fibronectin Expression in Mesangial Cells via Inhibiting cFLIP," PLoS One, vol. 9, no. 4, p. e93588, 2014.

[79] X. Li, Z. Xu, S. Wang et al., "Emodin ameliorates hepatic steatosis through endoplasmic reticulum-stress sterol regulatory element-binding protein 1c pathway in liquid fructose-feeding rats," Hepatology Research, vol. 46, no. 3, pp. E105-E117, 2016.

[80] X. Li, N. Mo, and Z. Li, "Ginsenosides: potential therapeutic source for fibrosis-associated human diseases," Journal of Ginseng Research, vol. 44, no. 3, pp. 386-398, 2020.

[81] S. S. Patel, A. Acharya, R. S. Ray, R. Agrawal, R. Raghuwanshi, and P. Jain, "Cellular and molecular mechanisms of curcumin in prevention and treatment of disease," Critical Reviews in Food Science and Nutrition, vol. 60, no. 6, pp. 887-939, 2020.

[82] X. Sun, Y. Liu, C. Li et al., "Recent Advances of Curcumin in the Prevention and Treatment of Renal Fibrosis," BioMed Research International, vol. 2017, Article ID 2418671, 9 pages, 2017. 
[83] R. Jakhar, S. Paul, A. K. Chauhan, and S. C. Kang, "Morin hydrate augments phagocytosis mechanism and inhibits LPS induced autophagic signaling in murine macrophage," International Immunopharmacology, vol. 22, no. 2, pp. 356-365, 2014.

[84] M. P. Singh, R. Jakhar, and S. C. Kang, "Morin hydrate attenuates the acrylamide-induced imbalance in antioxidant enzymes in a murine model," International Journal of Molecular Medicine, vol. 36, no. 4, pp. 992-1000, 2015.

[85] K. Sinha, J. Ghosh, and P. C. Sil, "Morin and its role in chronic diseases," Advances in Experimental Medicine and Biology, vol. 928, pp. 453-471, 2016.

[86] J. Gu, C. Chen, J. Wang et al., "Withaferin A Exerts Preventive Effect on Liver Fibrosis through Oxidative Stress Inhibition in a Sirtuin 3-Dependent Manner," Oxidative Medicine and Cellular Longevity, vol. 2020, Article ID 2452848, 17 pages, 2020.

[87] S. Rayees and F. Malik, "Withania somnifera: from traditional use to evidence based medicinal prominence," in Science of Ashwagandha: Preventive and Therapeutic Potentials, pp. 81103, Springer, Cham, 2017.

[88] S. H. Kim, E. R. Hahm, K. B. Singh, S. Shiva, J. Stewart-Ornstein, and S. V. Singh, "RNA-seq reveals novel mechanistic targets of withaferin A in prostate cancer cells," Carcinogenesis, vol. 41, no. 6, pp. 778-789, 2020.

[89] C. S. Peddakkulappagari, M. A. Saifi, A. Khurana, P. Anchi, M. Singh, and C. Godugu, "Withaferin A ameliorates renal injury due to its potent effect on inflammatory signaling," BioFactors, vol. 45, no. 5, pp. 750-762, 2019.

[90] J. Tian, Y. Liu, and K. Chen, "Ginkgo biloba extract in vascular protection: molecular mechanisms and clinical applications," Current Vascular Pharmacology, vol. 15, no. 6, pp. 532-548, 2017.

[91] A. S. P. Pereira, A. J. Banegas-Luna, J. Peña-García, H. PérezSánchez, and Z. Apostolides, "Evaluation of the AntiDiabetic Activity of Some Common Herbs and Spices: Providing New Insights with Inverse Virtual Screening," Molecules, vol. 24 , no. $22,2019$.

[92] M. N. Boukhatem, M. A. Ferhat, A. Kameli, F. Saidi, and H. T. Kebir, "Lemon grass (Cymbopogon citratus) essential oil as a potent anti-inflammatory and antifungal drugs," Libyan Journal of Medicine, vol. 9, no. 1, p. 25431, 2014.

[93] X. Zhang, Y. Cheng, Q. Zhou et al., "The Effect of Chinese Traditional Medicine Huaiqihuang $(\mathrm{HQH})$ on the Protection of Nephropathy," Oxidative Medicine and Cellular Longevity, vol. 2020, Article ID 2153912, 10 pages, 2020. 